\title{
Megadolicocolon de presentación poco usual en un paciente de edad avanzada: a propósito de un caso
}

\author{
Megadolicocolon with unusual presentation in an elderly patient: \\ a case report
}

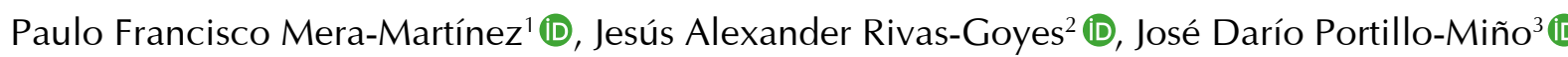

1 Médico, residente de Cirugía General, Universidad Nacional de Colombia, Bogotá, D.C., Colombia. Departamento de Cirugía, Hospital Universitario Departamental de Nariño, Pasto, Colombia.

2 Médico, especialista en Cirugía General, Escuela Latinoamericana de Medicina, La Habana, Cuba. Departamento de Cirugía, Hospital Universitario Departamental de Nariño, Pasto, Colombia.

3 Médico, Fundación Universitaria San Martin; Departamento de Investigación, Hospital Infantil Los Ángeles, Pasto, Colombia.

Póster presentado en el 45 Congreso "Semana Quirúrgica Nacional", Foro Quirúrgico Colombiano, 19 a 21 de agosto de 2019 , Bogotá, D.C., Colombia.

\section{Resumen}

El megadolicocolon es una patología inusual, de etiología no bien esclarecida. Tal parece ser de origen congénito, se presenta con frecuencia en recién nacidos y lactantes con vólvulos de colon. Su incidencia es variable y se encuentra entre 1,9 y $28,5 \%$.

Este reporte de caso es excepcional debido a que se presenta con una evolución y comportamiento agresivo de la enfermedad, pues se trata de un paciente de edad avanzada, con antecedente de sigmoidopexia y cuadro clínico de 7 meses de evolución consistente en estreñimiento crónico y exacerbación difusa de dolor abdominal incapacitante, quién requirió procedimiento quirúrgico de emergencia para su manejo.

Palabras clave: colon; megacolon; vólvulo intestinal; abdomen agudo.

\begin{abstract}
Megadolichocolon is an unusual pathology of not well clarified etiology. It seems to be of congenital origin and occurs frequently in newborns and infants with colon volvulus. Its incidence varies between $\mathrm{I} .9 \%$ and $28.5 \%$. This case report is exceptional because it presents with an aggressive evolution and behavior of the disease, since it
\end{abstract}

Fecha de recibido: 12/12/2019 - Fecha de aceptación: 28/01/2020

Correspondencia: Paulo Francisco Mera Martínez, Hospital Universitario Departamental de Nariño, Calle 22 \# 7-93, segundo piso, oficina de Cirugía, Pasto, Colombia. Teléfono: (+57) 3217758252

Correo electrónico: paulofrancisco4@hotmail.com

Citar como: Mera-Martínez PF, Rivas-Goyes JA, Portillo-Miño JD. Megadolicocolon de presentación poco usual en un paciente de edad avanzada: a propósito de un caso. Rev Colomb Cir. 2020;35:695-8. https://doi.org/10.30944/20117582.487

Este es un artículo de acceso abierto bajo una Licencia Creative Commons - BY-NC-ND https://creativecommons.org/licenses/by-ncnd/4.0/deed.es 
is an elderly patient, with a history of sigmoidopexy and a clinical evolution of 7 months consisting of chronic constipation and diffuse exacerbation of disabling abdominal pain, who required an emergency surgical procedure for its management.

Keywords: colon; megacolon; intestinal volvulus; abdomen, acute.

\section{Introducción}

El megadolicocolon ha sido descrito como una variante en el desarrollo y se caracteriza por la redundancia del colon. El incremento en la longitud del colon fue descrito por primera vez en I82O por Monterossi mediante una autopsia ${ }^{\mathrm{I}}$. Con el advenimiento de la tomografía computarizada (TC), Phillips y colaboradores reportaron la prolongación profusa del intestino con alteraciones en la movilidad de los segmentos ascendentes y descendentes ${ }^{2}$.

El dolicocolon se define por el incremento de la longitud del asa del colon sigmoide que pasa por encima de la línea que une las crestas iliacas, del colon transverso que pasa por debajo de la misma línea, y de asas adicionales en la flexión hepática y esplénica ${ }^{3}$. Existe variabilidad entre los estudios para determinar su epidemiología. Moeller et al., ${ }^{4}$ describieron el colon redundante en 18 pacientes de 744 casos para una incidencia aproximada de 2,4 \%, mientras que Kantor et al., ${ }^{5}$ en 1934 reportaron 258 casos de I6I4 pacientes quienes fueron diagnosticados mediante radiografías con una incidencia del $16 \%$, Bryant et al., ${ }^{6}$ informaron una incidencia del $14 \%$ de 242 cadáveres examinados y Larimore et al., ${ }^{7}$ encontraron una incidencia de $28,5 \%$. La redundancia del colon parece ocurrir a cualquier edad, en ambos sexos y sin especial preferencia por algún tipo de comportamiento ${ }^{8}$. Además, cabe resaltar que las manifestaciones principales se exhiben en edad temprana y rara vez en población adulta ${ }^{9}$.

\section{Caso clínico}

Se trata de un paciente masculino de 55 años de edad, con antecedente de devolvulación del colon y cuadro clínico de 7 meses de evolución consistente en estreñimiento crónico y exacerbación de dolor abdominal difuso e incapacitante, que lo obligó a consultar al servicio de Urgencias, donde ingresa con inestabilidad hemodinámica y signos de defensa abdominal. Se decidió llevar a procedimiento quirúrgico de emergencia por sospecha clínica de abdomen agudo secundario a obstrucción intestinal y distensión abdominal marcada, aunque sin hallazgos imagenológicos sugestivos de la enfermedad.

Se practicó laparotomía mediana por incisión previa y se encontraron múltiples adherencias peritoneales, doble vólvulo del colon izquierdo, vólvulo de colon sigmoides con gran distensión, signos isquémicos y distensión tóxica de colon descendente, colon transverso con meso largo y colon prominente, que también presenta vólvulo y dolicocolon de forma incidental. Fue imperativo liberar adherencias múltiples con disección cortante, resecar desde el tercio proximal del colon transverso hasta la unión rectosigmoidea y dejar colostomía en flanco izquierdo (figura I).

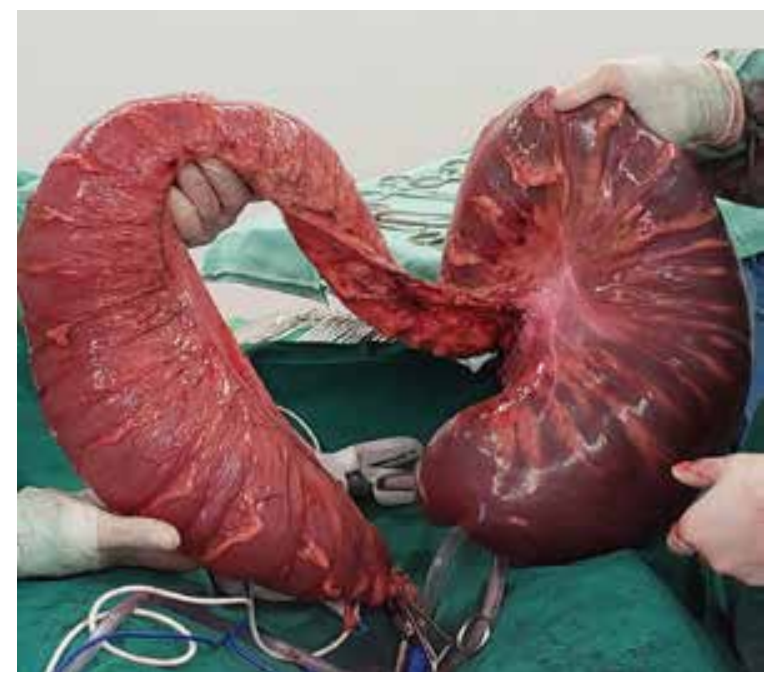

Figura 1. Se observa megadolicocolon con áreas hemorrágicas y necrosis en varios segmentos y distensión importante en la resección quirúrgica completa.

Fuente: Los autores. 
Se trasladó a la Unidad de Cuidados Intensivos para manejo integral y soporte hemodinámico y fue dado de alta quince días después, sin complicaciones.

El reporte de patología a nivel macroscópico refiere un segmento de colon que mide $145 \mathrm{~cm}$ de longitud por $5 \mathrm{~cm}$ de diámetro en los bordes de sección, con marcada dilatación, llegando a medir aproximadamente $15 \mathrm{x} \mathrm{I} 7 \mathrm{~cm}$ en el tercio medio, con mucosa hemorrágica de forma profusa al corte. En la descripción microscópica se observan los bordes de sección con marcado edema, congestión vascular, hemorragia y necrosis de la mucosa; en la serosa de manera focal se observa reacción de células gigantes tipo cuerpo extraño, más tejido adiposo maduro con congestión y focos de hemorragia, hallazgos compatibles con necrosis hemorrágica, marcada dilatación de la pared colónica y vólvulo intestinal.

\section{Discusión}

El megadolicocolon es una patología predominante en etapas tempranas de la vida, cuya sospecha clínica en edad avanzada requiere de un conocimiento y comprensión preciso de la enfermedad. Se ha considerado al dolicosigma, megadolicosigma, dolicocolon y megadolicocolon, como estadios de algún defecto y la alteración en la función evacuante es la base de esta malformación ${ }^{10}$.

En cuanto a la prevalencia de megadolicocolon, dolicocolon o megacolon en Colombia, no hay datos suficientes que nos permitan abordar sistemáticamente está información. En Brasil, se ha descrito la ocurrencia del dolicocolon sin megacolon en pacientes con enfermedad de Chagas en un $\mathrm{I}_{4}, 8 \%$ " . A diferencia, la región del suroccidente colombiano no es área endémica de Chagas y, por consiguiente, no es un factor condicionante de la patología en nuestro caso. Es paradójico encontrar dolicocolon y megacolon en el mismo paciente, lo que hace este caso aún más interesante.

Para el enfoque clínico inicial, se ha identificado una tríada clásica que consiste en estreñi- miento, dolor abdominal y distensión ${ }^{12,13}$. Puede asemejarse a otras patologías quirúrgicas como la enfermedad de Hirschsprung, apendicitis aguda, gastroduodenitis, obstrucción intestinal crónica y enfermedad tumoral en el colon ${ }^{14}$. El diagnóstico definitivo del megadolicocolon se establece mediante enema de bario o colonografía por tomografía computarizada ${ }^{15}$.

Existen varios métodos terapéuticos que permiten un tratamiento más conservador en caso de vólvulo o estreñimiento según las directrices de la Asociación Americana de Gastroenterología, a pesar de que no tienen una eficacia del Ioo $\%{ }^{16}$. Por lo tanto, deben emplearse los elementos de juicio necesarios para decidir si un abordaje quirúrgico es pertinente cuando los síntomas son refractarios y las complicaciones ocasionan emergencias quirúrgicas, como en el caso presentado. El grupo de Lane et al., ${ }^{17}$ quienes practicaron colectomía para constipación crónica, reportó una tasa de mortalidad que alcanzó el 20,5\%, mientras otros autores han ejecutado resecciones de segmentos del colon o hemicolectomías con resultados más alentadores ${ }^{13,18-20}$.

Durante las últimas décadas, la cirugía colorrectal ha pasado varias etapas incluyendo, desde la realización de resecciones segmentarias del sigmoide, la resección de dolicosigma y la hemicolectomía del lado izquierdo, hasta la expansión de las resecciones como la colectomía subtotal y total ${ }^{21,22}$. Sin embargo, todavía no se han definido indicaciones precisas para el tratamiento quirúrgico.

En Colombia, no hay literatura que describa un contexto quirúrgico del megadolicocolon con las características mencionadas. Un estudio realizado por Hequera et al., ${ }^{23}$, pone de manifiesto los factores condicionantes para la impactación fecal, entre los cuales cabe destacar la senectud $(42,52 \%)$, trastornos neurológicos (20,69\%), dolicocolon $(9,19 \%)$ y megacolon por Chagas $(5,74$ $\%)$. De este grupo, sólo 3 pacientes fueron intervenidos quirúrgicamente mediante colostomía transversa y una cecostomía ${ }^{23}$. 


\section{Conclusiones}

La presentación del megadolicocolon con las características descritas es lo usual, sin embargo, su ocurrencia en la práctica clínica es excepcional y potencialmente fatal en un escenario cuyas complicaciones plantean un contexto desafiante en el diagnóstico y tratamiento quirúrgico temprano; ya que, si se retrasa, puede afrontar circunstancias deletéreas que influencian significativamente en el desenlace del paciente.

\section{Consideraciones éticas}

Consentimiento informado: El paciente ha declarado mediante consentimiento informado la autorización para publicar el reporte de caso y las imágenes correspondientes.

Conflicto de interés: Los autores declaran no poseer ningún conflicto de intereses.

Fuente de financiación: Autofinanciado por los autores.

\section{Referencias}

I. Monterossi P. Uber widernatürlische Biegungen des Dickdarms als Uhrfache des Todes neugeborener Kinder. Deutsch Arch Physiol. 1820;6:556-7I.

2. Phillips M, Patel A, Meredith P, Will O, Brassett C. Segmental colonic length and mobility. Ann R Coll Surg Engl. 2015;97:439-44.

https://doi.org/IO.I308/0035884I5XI4I8I254790527

3. Caffey J. The large intestine. In: Caffey J, editor. Pediatric X-ray diagnosis. Chicago: Book Medical Publishers Inc; I96I. p. 6I3-46.

4. Moeller PF. The redundant colon. Acta Radiol. I926;6:432-57. https://doi.org/IO.3IO9/000I6922609139505

5. Kantor JL. Common anomalies of the duodenum and colon: Their practical significance. JAMA. 1931;97:17859o. https://doi.org/IO.IOoI/jama.I93I.02730240035008

6. Bryant J. Observations upon the growth and length of the human intestine. Am J Med Sci. 1924;167:499-520. https://doi.org/I0.I097/0000044I-192404000-00003

7. Larimore JW. The human large intestine in the newborn and in the adult. Ann Clin Med. 1926;5:439-63.

8. Galambos A, Galambos WM. Redundancy of the colon. Am J Dig Dis. 1946;13:87-IOI. https://doi.org/IO.IOO7/BF03002757

9. Yik YI, Cook DJ, Veysey DM, Tudball CF, Cain TM, Southwell BR, et al. How common is colonic elongation in children with slow-transit constipation or anorectal retention? J Pediatr Surg. 20I2;47:I4I4-20. https://doi.org/IO.IOI6/j.jpedsurg.2012.0I.007

IO. Black CE. Displacements of the colon. Ann Surg. I9I2;56:888-99.

https://doi.org/IO.I097/00000658-I9I2I2000-000II

II. Castro C, Hernandez EB, Rezende J, Prata A. Occurrence of dolichocolon without megacolon in chronic Chagas disease patients. Rev Soc Bras Med Trop. 20I2;45:353-6. https://doi.org/I0.1590/So037-868220120003000I4

I2. Brumer P, Seppälä P, Wegelius U. Redundant colon as a cause of constipation. Gut. 1962;3:I40-I. https://doi.org/IO.II36/gut.3.2.I40

I3. Hollender LF, Muller AM, Viville C, Santizo G. Dolichocolon in adults: surgical indications. Acta Gastroenterol Belg. 1965;28:550-67.

I4. Tomita R, Howard ER. Clinical studies on anorectal myectomy for chronically constipated patients with outlet obstruction in childhood. Hepatogastroenterology. 2008;55:I600-5.

I5. Hanson ME, Pickhardt PJ, Kim DH, Pfau PR. Anatomic factors predictive of incomplete colonoscopy based on findings at CT colonography. AJR Am J Roentgenol. 2007;I89:774-9. https://doi.org/IO.22I4/AJR.07.2048

I6. Bharucha AE, Dorn SD, Lembo A, Pressman A. American Gastroenterological Association medical position statement on constipation. Gastroenterology. 2013;I44:2II-7. https://doi.org/IO.I053/j.gastro.2012.I0.029

I7. Lane WA. Remarks on the results of the operative treatment of chronic constipation. Br Med J. I908;1:I26-30.

I8. Slesinger EG. Elongation of the pelvic colon and its surgical treatment. Lancet. 1926;2:274-5.

I9. Davis DA. A new concept in the treatment of symptomatic redundant colon or dolichocolon. West J Surg Gynecol. 1960;68:IOI-6.

2o. Han EC, Oh HK, Ha HK, Choe EK, Moon SH, Ryoo SB, et al. Favorable surgical treatment outcomes for chronic constipation with features of colonic pseudo-obstruction. World J Gastroenterol. 20I2;I8:444I-6. https://doi.org/I0.3748/wjg.vi8.i32.444I

2I. Lundin E, Karlbom U, Påhlman L, Graf W. Outcome of segmental colonic resection for slow-transit constipation. Br J Surg. 2002;89:1270-4. https://doi.org/IO.IO46/j.1365-2168.2002.02213.x

22. Raahave D, Loud FB, Christensen E, Knudsen LL. Colectomy for refractory constipation. Scand J Gastroenterol. 2010;45:592-602. https://doi.org/I0.3109/00365521003587796

23. Hequera JA, Obregón JG, Cabas J, Toresan L. Impactación fecal. Rev Argent Coloproctología. 2012;23:194-9. 